\title{
Incompatibility, Inconsistency, and Logical Analysis in Tractatus Logico-Philosophicus
}

\author{
Ivan Welty
}

Received: date / Accepted: date

\begin{abstract}
Statements of degree appear to falsify basic doctrines in Tractatus LogicoPhilosophicus. I offer a fresh formulation of the challenge and assess a solution proposed on Wittgenstein's behalf by Sarah Moss. I find that Moss's proposal fails. The proposal rides in part on novel interpretations of pronouncements by Wittgenstein on the nature of the elementary proposition. I find that the interpretations cannot be sustained but that Moss's textual case hints at important and overlooked features of the Tractarian program. I develop Wittgenstein's comparison of propositions to measuring instruments and apply it throughout, showing that it captures philosophical commitments in Tractatus that otherwise tend to slip from view.
\end{abstract}

Keywords Color incompatibility problem; elementary propositions; logical analysis; logical space; rational reconstruction; simples; solipsism; Tractatus; Wittgenstein

\section{Problem}

Frege and Russell gave probing accounts of the proposition but left basic philosophical questions unsettled. What exactly is the relationship between a squiggle and another part of the world when the former is true or false depending on how things stand with the latter? How do two squiggles, or a squiggle and a sound, lay down the same truth-condition? How can the truth of one squiggle follow directly from the truth of another squiggle laying down what is apparently a different truth-condition? Frege and Russell made advances on these and related questions in framing their logical apparatuses, but questions on which definite advances could be made by applying and retooling their apparatuses quickly dominated their attention. In Tractatus LogicoPhilosophicus, Wittgenstein tried for a ground-up account of the proposition and all matters directly connected to it that left no philosophical issue in shadow unless as a

Ivan Welty

Philosophy Department, Willamette University, 900 State Street, Salem, OR 97301, USA,

E-mail: iwelty@willamette.edu 
matter of principle it had to remain so. The construction and fruitful application of a logical apparatus was not his primary aim.

On a defensible telling of the story, Wittgenstein starts by conceiving the proposition as a fact modeling other facts. This immediately prompts two questions. First, how are the basic constituents of the model correlated with the basic constituents of the fact that it models? Second, how are the structures of the two facts correlated? The first correlation is supposed to be effected by convention: we assign a name to an object so that the name can serve as the object's proxy (Wittgenstein (1998) 3.22, 3.315, passim; henceforth I cite Tractatus by decimal number alone). The second correlation is supposed to be effected by the identification of the logical forms in the two facts $(2.2,4.12$, passim). The logical form of a fact is the space of possible combinations of the basic constituents comprising it. A structure is a point in a space of possible combinations.

Tractatus embeds these preliminaries in a densely interconnected network of philosophical commitments. Two commitments concern propositions that Wittgenstein characterizes as elementary, which for the moment we may take to mean that they are truth-functionally uncompounded:

(i) Every elementary proposition is logically independent of the others, that is, no elementary proposition or its negation can be logically inferred from any other elementary proposition or its negation. (4.211, 2.06, passim)

(ii) Every non-elementary proposition is a truth-functional combination of elementary propositions. (5.3)

Attributions of color, of which there are typically more than two available, give no obvious sign of being truth-functional compounds, yet they are necessarily incompatible while elementary propositions are supposed to be logically independent. What gives?

If (i) and (ii) were Wittgenstein's only relevant commitments, he could allow that color attributions are elementary and that elementary propositions can be necessarily incompatible without being logically inconsistent (henceforth by 'incompatible' I will mean necessarily incompatible and by 'inconsistent' I will mean logically inconsistent). But Wittgenstein also holds that

(iii) All necessity is logical. $(6.37,6.375)$

Wittgenstein's idea is that all necessary relations, prima facie material or otherwise, trace to how propositions are compounded truth-functionally of elementary propositions, in other words, are formal. Combining (iii) with (i) (ii) renders color attributions a hard puzzle. Similar puzzles arise with respect to brightness, pitch, and other matters of degree, but by custom they are referred to collectively as the color incompatibility problem 1

\footnotetext{
1 A further word concerning Wittgenstein's lightly-sketched logical apparatus is in order. Wittgenstein extended truth-tables to a semantics for quantified formulas by admitting truth-functions with infinitely many arguments. For a contemporary account, see Frascolla (2007). Rogers and Wehmeier (2012) show that the primary notational constraints laid down in Tractatus - that compound formulas involve only the $\mathrm{N}$-operator, and that identity be expressed by sameness of name rather than via an equation - are jointly satisfiable by a formal language equal in expressive power to first-order logic. Miller (1995) supplies a Tractarian situation semantics with a truth definition materially equivalent to Tarski-style truth definitions.
} 
Tractatus gestures toward the possibility of logical analyses on which color attributions come out as truth-functional compounds of elementary propositions, but Wittgenstein gave up hope for such an analysis on his return to philosophy in 1929. Still the prospect of an analysis of color attributions consistent with Tractarian commitments - in short, a solution to the color incompatibility problem - continues to tantalize philosophers. In what follows, I frame the color incompatibility problem on fresh lines before assessing a solution proposed by Moss (2012) (with Carruthers (1990) as an acknowledged precedent). I find that Moss's proposal fails. The proposal rides in part on novel interpretations of pronouncements by Wittgenstein on the nature of the elementary proposition. I find that the interpretations cannot be sustained but that Moss's textual evidence hints at important and overlooked features of the Tractarian program. Careful assessment of Moss's proposal thus helps to restore the color incompatibility problem to the philosophical setting that gives it significance.

\section{Logical Analysis}

No proposed solution to the color incompatibility problem can be assessed without a firm grasp of the Tractarian basics, including commitments that constrain the range of admissible logical analyses. We will gain a firmer grasp of the relevant basics by developing Wittgenstein's comparison of propositions to measuring instruments (2.1511-2.15121).

A phase space is a geometrical model of the possible states of a physical system. To each degree of freedom of the system there corresponds a parameter (dimension) of the phase space, so that to each state of the system there corresponds a point in the phase space. As an example, we can model a system comprising the colors blue, green, red, and yellow as a one-parameter, four-point phase space. Of special interest are phase spaces, called by Wittgenstein logical spaces, whose parameters are propositions taking values in $\{\mathrm{T}, \mathrm{F}\}$. Sets of points in logical space - or, as we will say, regions (possibly empty) of logical space - are truth-functions, and truth-functions are entailed by their subregions (5.12-5.121) (see figure 1). A proposition is elemen-

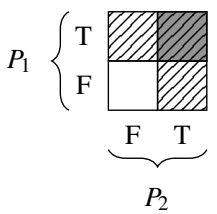

Fig. 1 Logical space with parameters $P_{1}$ and $P_{2}$; truth-function $P_{1} \vee P_{2}$ indicated by hatching and $P_{1} \wedge P_{2}$ by shading

tary if the fact that exists when the proposition is true is atomic (4.25). An atomic fact is a 'combination of objects' (2.01) and an object is 'the fixed, the existent' (2.0271). There will be more to say about atomic facts and objects shortly.

If the points in a phase space $\mathscr{S}$ are pairwise incompatible, by (iii) they must be inconsistent. Tractarian logical analysis of $\mathscr{S}$ will yield a logical space $\mathscr{L}$ such 
that to distinct points in $\mathscr{S}$ there correspond disjoint regions in $\mathscr{L}$. Conversely, we can think of $\mathscr{S}$ as a coarse-graining of $\mathscr{L}$, on analogy with the method of grouping physical microstates that are indistinguishable relative to macrostate parameters ${ }^{2}$ If the logical space yielded by a logical analysis has all of its parameters elementary, the analysis is complete; otherwise, it is incomplete. Every proposition, and by extension every logical space, is subject to complete analysis (3.2, 3.205, 5, passim). A logical space might have more than one course-graining, as the possibility of incomplete analysis already shows.

Logical analysis yields a logical space, but it is not yet clear what counts as logical analysis. Wittgenstein's basic idea is that logical analysis unfolds definitions. Every analysis is expressible in a definition (3.24), and in turn definition 'shows the way' to an analysis (3.261) 3 Naturally, given that analysis unfolds definitions, 'there is one and only one complete analysis of the proposition' (3.25). By extension, there is one and only one complete analysis of logical space. Wittgenstein identifies logically equivalent propositions (5.14ff). Coarse-graining preserves entailment relations, in other words, a subregion of a region is coarse-grained by a subregion of the coarsegraining of the region 4

We conclude with a simple illustration of logical analysis. The two propositions of my idiolect, ' $a$ is single' and ' $a$ is wedded', are incompatible, hence there must be a logical analysis that exposes them as inconsistent (the reason for the restriction to my idiolect will be made clear in $\$ 5$. By my definitions, $a$ is wedded just in case ' $a$ is married' is true and single just in case it is false. An analysis proceeding in accordance with other definitions will be of something other than these propositions. The analysis is complete if ' $a$ is married' is elementary and incomplete otherwise. A solution to the color incompatibility problem will be an analysis of color attributions on these lines, unfolding color space into logical space via definitions. Note that while the cardinality of a coarse-graining (in the present sense) might be equal to that of the space it coarse-grains, as in this example, it also might be smaller, as when an affirmation of the sibling relation analyzes into a disjunction of affirmations of gendered relations.

\section{Moss's Proposal}

We now examine Moss's proposed solution to the color incompatibility problem, generalizing as Moss does from an example. Consider a color space $\mathscr{C}$ comprising blue, green, red, and yellow (in attribution to a given object, which we allow to remain

\footnotetext{
2 See Ridderbos 2002) p. 67-70 for a historically-informed explication of coarse-graining. Coarsegraining is now a standard technique in multiscale modeling across the natural sciences and mathematics, useful, e.g., when measurement uncertainty precludes finer-grained discrimination and in abstracting simplified subsystems.

3 Wittgenstein rules out idle definition as not genuinely definition (3.328).

4 In other notation, if the coarse-graining is a surjection $\psi: \mathscr{L} \rightarrow \mathscr{S}$ then for sets $X$ and $Y$ of points in $\mathscr{L}, X \subseteq Y \Rightarrow \psi[X] \subseteq \psi[Y]$, where $\psi[X]=\{\psi(x): x \in X\}$.
} 
implicit). Fix a bijection from $\mathscr{C}$ to the set of binary strings of length two-say,

$$
\begin{aligned}
\text { blue } & \mapsto 00 \\
\text { green } & \mapsto 01 \\
\text { yellow } & \mapsto 10 \\
\text { red } & \mapsto 11 .
\end{aligned}
$$

Let $Q_{i}$ be the set of strings with 1 in the $i$ th place. Regarded as a set of possible worlds, each $Q_{i}$ is a proposition and logically independent of the other. Now consider the logical space of which these propositions are the parameters. By construction of the parameters, there is a correspondence between points in $\mathscr{C}$ and points in the logical space (see figure 2). As the correspondence is stipulated (definitional), incompatibility has been reduced to inconsistency.

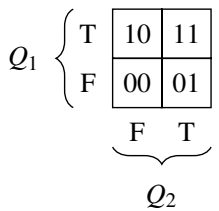

Fig. 2 Moss-style analysis of $\mathscr{C}$

Moss's proposal generalizes straightforwardly to spaces of cardinality $2^{n}$ for $n>2$ and beyond them to spaces with continuum-many points. Moss generalizes to arbitrary finite cardinalities and arbitrary necessary relations as follows. Let arbitrarily many propositions be given, with arbitrary necessary relations between them. Again identify each proposition with the set of possible worlds in which it is true. Form an Euler diagram of the propositions. Distinct zones in the diagram represent mutually incompatible propositions. If there are $2^{n}$ zones for some $n$, submit them to the procedure just outlined. If there are finitely many zones but not a power of two, partition zones until their total number reaches a power of two and then proceed as above. If there is no power of two between (inclusive) the number of zones in the Euler diagram and the number of underlying possible worlds then, as Moss recognizes, the strategy cannot be implemented 5

Whatever its merits, Moss's proposal falls short as a solution to the color incompatibility problem even when the number of incompatible propositions is a power of two. A solution to the problem will be a logical analysis of color attributions satisfying all Tractarian conditions. One condition, so far unmentioned here, concerns the relationship between a proposition and its negation. A Tractarian proposition 'shows how things stand, if it is true' (4.022). What this means in the case of a negative proposition is not immediately clear. Wittgenstein begins to clarify it when he writes

\footnotetext{
5 Moss does not use Euler diagrams but elaborates an equivalent procedure (p. 846). I have streamlined Moss's presentation in other minor ways. Moss specifies the propositions in the four-color case as 'that $A$ is red or yellow' and 'that $A$ is red or green', labeled $P_{1}$ and $P_{2}$, respectively (p. 844). Only for continuummany points does Moss label the propositions as I have done and interpose strings (p. 844-5).
} 
that to a proposition and its negation there 'corresponds one and the same reality' (4.0621). Further on he elaborates:

One could say, the denial is already related to the logical place determined by the proposition that is denied.

The denying proposition determines a logical place other than does the proposition denied.

The denying proposition determines a logical place, with the help of the logical place of the proposition denied, by saying that it lies outside the latter place. (4.0641)

Denial, logical addition, logical multiplication, etc., etc., are operations.

(Denial reverses the sense of a proposition.) (5.2341)

Simply put, $\neg P$ denies that $P$. More elaborately, if we compare a proposition to a unit magnitude (base unit, when elementary), the proposition's negation marks the scale's zero, as ' 0 meters' marks the zero of the meter scale 6

Now consider Moss's proposal ${ }^{7}$ Moss's propositions are sets of worlds. Construing negation standardly, as complementation, figure 2 not only illustrates a mapping between two spaces, it fixes propositional contents: $Q_{1}$ says that yellow-or-red is true, $\neg Q_{1}$ says that blue-or-green is true, $Q_{2}$ says that green-or-red is true, and $\neg Q_{2}$ says that blue-or-yellow is true. On this approach, $\neg Q_{1}$ (likewise, mutatis mutandis, $\neg Q_{2}$ ) does not correspond to 'one and the same reality' as does $Q_{1}$, does not determine a logical place 'by saying that it lies outside' of the place determined by $Q_{1}$, in short, does not deny that $Q_{1}$. We might suppose that $\neg Q_{1}$ is the Tractarian negation of $Q_{1}$ given the occurrence in ' $\neg Q_{1}$ ' of the expressions ' $\neg$ ' and ' $Q_{1}$ ', but $\neg Q_{1}$ has been fixed by stipulation, not by an operation on $Q_{1}$. Moss's proposal does not meet Tractarian conditions on negation.

Moss's proposal cannot be made to meet the conditions by simply reinterpreting ' $\neg$ ' on Tractarian lines. The Tractarian negations of yellow-or-red and green-orred are not-yellow-and-not-red and not-green-and-not-red, respectively. Blue does not follow logically from the conjunction of not-yellow-and-not-red and not-green-andnot-red, although on Moss's proposal it is supposed to follow from $\neg Q_{1} \wedge \neg Q_{2}$. Blue is logically consistent with the conjunction of not-yellow-and-not-red and not-greenand-not-red, but it is also logically consistent with (resorting to logical notation, interpreted on Tractarian lines) $Q_{1} \wedge Q_{2}, Q_{1} \wedge \neg Q_{2}$, and $\neg Q_{1} \wedge Q_{2}$. If in Moss's proposal we interpret ' $\neg$ ' on Tractarian lines, the necessary relations among red, yellow, green, and blue do not match up with the logical relations among $Q_{1} \wedge Q_{2}, Q_{1} \wedge \neg Q_{2}$, $\neg Q_{1} \wedge Q_{2}$, and $\neg Q_{1} \wedge \neg Q_{2}$.

Wittgenstein was prepared to relax his previous commitments when he took up the color incompatibility problem on his return to philosophy, but relaxing Tractarian conditions on negation does not salvage Moss's proposal. Let us grant that figure 2 fixes propositional contents. If $Q_{1}$ and $\neg Q_{1}$ - that is, yellow-or-red and blue-or-green, respectively - necessarily exclude each other, this owes to the incompatibility of each

\footnotetext{
6 Wittgenstein compares the negation of a proposition to the zero-point of a scale in discussions with members of the Vienna Circle in the winter of 1929-30 (Wittgenstein (1979, p. 67 and p. 86).

7 I am grateful to an anonymous reviewer of this journal for criticism that helped me to clarify the argument in this and the following three paragraphs.
} 
of yellow and red with each of blue and green. If $Q_{1} \wedge Q_{2}$ - that is, yellow-or-red conjoined with green-or-red - amounts to the attribution of just red, this owes to the incompatibility of red with each of yellow and green (and blue). Stipulating propositional contents in accordance with figure 2 reduces inconsistency to incompatibility, not the other way around.

Like negation in Moss's proposal, Tractarian negation amounts to complementation, but over regions of logical space, not sets of worlds. Logical space meets conditions that a domain of worlds need not, including (i) (iii). Tractarian negation plays directly on the resulting structure. This is why the Tractarian negation of yellow-orred is, and can only be, not-yellow-and-not-red. On Moss's proposal, by contrast, the negation of yellow-or-red is determined by whatever other worlds there happen to be, a matter that Moss's proposal does not make subject to logic 8

We do not have a Tractarian analysis of color to contrast with Moss's proposal, but if we admit as satisfactory the Tractarian analysis of wedded/single space in $\$ 2$ a concrete illustration of the difference between Moss-style analysis and Tractatusstyle analysis is available. We reduced the incompatibility between ' $a$ is wedded' and ' $a$ is single' to the inconsistency between ' $a$ is married' and 'it is not the case that $a$ is married'. A Moss-style analysis might stipulate that $P$ is true just in case $a$ is wedded and $\neg P$ is true just in case $a$ is single ${ }^{9}$ We can expand the example to four points by incorporating two further possibilities: $a$ might be not only wedded or single but also engaged or widowed, where all four points are understood to be pairwise incompatible and for simplicity we neglect the possibilities of divorce and remarriage. A Moss-style analysis of this space might stipulate that $P_{1}$ is true just in case $a$ is wedded or single, $\neg P_{1}$ is true just in case $a$ is engaged or widowed, $P_{2}$ is true just in case $a$ is widowed or single, and $\neg P_{2}$ is true just in case $a$ is engaged or wedded. By contrast, Tractarian parameters spanning the space might be that $a$ has been party to a marriage contract and that $a$ is foreseeably to be party to a marriage contract. On this analysis, $a$ is single if $a$ has not been party to a marriage contract and is not foreseeably to be party to a marriage contract, $a$ is wedded if $a$ has been party to a marriage contract and is foreseeably to be party to a marriage contract, $a$ is engaged if $a$ has not been party to a marriage contract but is foreseeably to be party to a marriage contract, and $a$ is widowed if $a$ has been party to a marriage contract but is not foreseeably to be party to a marriage contract.

\section{Elementarity}

Moss frames the significance of the color incompatibility problem as follows: 'If we cannot analyze color propositions into logically independent components, then a for-

\footnotetext{
8 Cf. 3.42. Moss passes lightly over the differences between Tractarian propositions and sets of worlds: ' $[\mathrm{t}]$ he ways in which genuine Tractarian propositions differ from sets of worlds are incidental to my discussion of the color incompatibility problem' (p. 842).

9 More fully, map wedded to 1 and single to 0 , then let $P$ be the singleton set $\{1\}$ and $\neg P$ be the singleton set $\{0\}$. The content of $\neg P$ is not fixed by an operation on the content of $P$. It is as though we laid it down that $0 u=1 u^{\prime}$ for units $u$ and $u^{\prime}$ : generally speaking this will not determine a conversion factor between $u$ and $u^{\prime}$. Note that the number of incompatible propositions in this example is two, that is to say, Moss's proposal falls short even before we reach statements of degree.
} 
tiori we cannot completely analyze all propositions, and we must abandon the central program of the Tractatus' (Moss (2012), p. 844). This is correct. However, Moss not only delivers (what is supposed to be) an analysis 'into logically independent components' but declares the components - the propositions $Q_{i}$ - to be elementary. This suggests that the bar a solution must clear has been raised from merely analyzing color attributions to analyzing them completely. One now naturally worries that the propositions $Q_{i}$ 'do not have the right form to be elementary' (p. 848). Moss anticipates the worry and offers that 'elementary color propositions may be gerrymandered disjunctions of simpler-looking color propositions, while all simpler-looking propositions are themselves non-elementary' (p. 844). In turn, this is supposed to be possible because Tractarian propositions are 'simple only relative to a system of description' (p. 849). Call this the relativity thesis. If it is true then Tractatus is due for wholesale reassessment, down to the principles that have been thought to generate the color incompatibility problem. Before embarking on wholesale reassessment, however, we will do well to examine Moss's textual evidence for the relativity thesis.

Wittgenstein compares systems of mechanics - more generally, 'systems for describing the world' - to coordinate systems or 'meshes' used in the description of planar figures. He adds that the world 'can be described more simply with one system of mechanics than with another' (6.342). These and surrounding pronouncements are commonly thought to develop, albeit impressionistically, some implications of core Tractarian doctrines for our understanding of natural science. Moss takes them for evidence of relativity. Close examination vindicates the common reading, and our discussion to this point enables us to sharpen it.

At 6.341, Wittgenstein explains what he means in the discussion of meshes by 'simple': 'It can happen that the description might have been simpler with the aid of a triangular mesh [than with the aid of a finer square mesh]; that is to say we might have described the surface more accurately with a triangular, and coarser, than with the finer square mesh.' The word here translated as 'simpler' is not elementarer but einfacher, elementar(sätze) being reserved in Tractatus for propositions asserting the existence of atomic facts ${ }^{10}$ Moreover, the 'surface' under description in Wittgenstein's analogy is not just any fact but itself a proposition (bild), and a system like Newton's mechanics is supposed to have among its purposes the imposition of a 'unified form'. All of this suggests that Wittgenstein's concern in these passages is not logical analysis but rational reconstruction, in broadly the sense familiar from later philosophy.

The suggestion is soon confirmed. Wittgenstein concludes 6.341 with the parenthetical remark:

\footnotetext{
10 The distinction between einfach and elementar is not clearly drawn in the notebooks but corresponding passages in Tractatus show that it eventually sharpened. For example,

Die empirische Realität ist begrenzt durch die Gesamtheit der Gegenstände. Die Grenze zeigt sich wieder in der Gesamtheit der einfachen Sätze. (Wittgenstein 1961) 26.4.16)

Die empirische Realität ist begrenzt durch die Gesamtheit der Gegenstände. Die Grenze zeigt sich wieder in der Gesamtheit der Elementarsätze. (Wittgenstein,1998) 5.5561)

In English: 'Empirical reality is limited by the totality of objects. The boundary appears again in the totality of simple/elementary propositions.'
} 
(As with the system of numbers [Zahlensystem] one must be able to write down any arbitrary number, so with the system of mechanics one must be able to write down any arbitrary physical proposition.)

The crucial condition on numeral systems is that they preserve factorizations ${ }^{11}$ When, therefore, Wittgenstein resumes by writing, 'And now we see the relative positions of logic and mechanics' (6.342), one naturally suspects that truth-functional structure, down to elementary propositions, is supposed to be preserved under change of mesh. The sentences that follow show that it is:

So too the fact that it can be described by Newtonian mechanics asserts nothing about the world; but this asserts something, namely, that it can be described in that particular way in which as a matter of fact it is described. The fact, too, that it can be described more simply [einfacher] by one system of mechanics than by another says something about the world.

If truth-functional structure down to elementary components varied with change of mesh, it certainly would assert something about the world that it can be described using Newtonian mechanics, just as, if numeral systems were restricted to different sets of irreducibles and their products, it would assert something about a number that it can be represented in a certain numeral system. What Wittgenstein calls "change of mesh' is the substitution of one coarse-graining for another coarse-graining of the same logical space. There is no reason to assume that one of them will be yielded by logical analysis of the other.

In 'Some Remarks on Logical Form', Wittgenstein begins reckoning in earnest with the color incompatibility problem. He considers and rejects an analysis of brightness that turns on distinct yet interchangeable units:

If ... we try to distinguish between the units and consequently write $E(2 b)=$ $E\left(b^{\prime}\right) \& E\left(b^{\prime \prime}\right)$, we assume two different units of brightness; and then, if an entity possesses one unit, the question could arise, which of the two $-b^{\prime}$ or $b^{\prime \prime}$ - it is; which is obviously absurd. (Wittgenstein (1929), p. 167-168)

According to Moss,

This passage shows that Wittgenstein does not reject his own gerrymandered analyses of color propositions because they contain logical constants. He rejects his attempted analyses because they do not work. This is evidence that Wittgenstein does not think that how we express a proposition in natural language is a reliable guide to whether it is a viable candidate for being an elementary proposition, in the sense of 'elementary' relevant to the central project of the Tractatus. (Moss (2012), p. 849)

Two paragraphs further on, Moss observes that Wittgenstein is 'sanguine about using apparently complex propositions to analyze apparently simple ones' and offers as

11 'Numeral system' is a more apt translation of Zahlensystem than Ogden's 'system of numbers' or Pears's and McGuinness's 'number-system'. Concerning a numeral system it makes sense to insist that 'one must be able to write down any arbitrary number', concerning a number system it does not. Note that Wittgenstein's analogy does not require that the numbers in question be uniquely factorizable, although if they are then the analogy to propositions is closer given his commitment to unique logical analyzability. 
the most plausible explanation of this fact Wittgenstein's putative commitment to relativity.

These interpretations are strained. In the passage from 'Some Remarks on Logical Form', Wittgenstein entertains an analysis of $E(2 b)$ on which $E\left(b^{\prime}\right) \wedge E\left(b^{\prime \prime}\right)$ is the analysans. He observes that $E(1 b)$ then forces a choice between $E\left(b^{\prime}\right)$ and $E\left(b^{\prime \prime}\right)$. These propositions must be logically equivalent yet distinct, an 'absurd' consequence given the identification of equivalent propositions. Wittgenstein remains sanguine about using a truth-functional compound to analyze something apparently simple for the unremarkable reason that analysis makes otherwise hidden structure explicit (4.002). I will say a bit more in the next section about what it means for structure to be hidden.

These are two key passages offered by Moss as evidence for the relativity thesis. A third passage demands separate treatment, as it involves very different issues 12

\section{Solipsism}

Moss quotes an exclamation from 1915 culminating seven weeks of reflection on the simplicity of objects: 'This object is simple for me!' (Wittgenstein (1961), 22.6.15henceforth entries in Wittgenstein (1961) are cited by date only). Insofar as objects make up atomic facts and atomic facts are described by elementary propositions, this exclamation does sound like possible evidence for the relativity thesis. To assess it, we will start with Wittgenstein's views on the subject (' $m e$ ') and work our way back to objects.

Wittgenstein holds that 'the subject does not belong to the world but it is a limit of the world' (5.632). Accordingly, he characterizes the subject only negatively (5.631).

12 A short survey of usages of the term 'elementary' in the literature surrounding Tractatus might be found useful. In Russell and Whitehead (1910), the term covers truth-functional compounds:

By an "elementary" proposition we mean one which does not involve any variables, or, in other language, one which does not involve such words as "all," "some," "the" or equivalents for such words. (p. 95)

So Russell and Whitehead can write, for example, 'If $p$ is an elementary proposition, $\sim p$ is an elementary proposition' $(* 1 \cdot 7$, p. 101). In a 1913 letter to Russell, Wittgenstein acknowledges their usage:

But the propositions of logic - and only they - have the property that their truth or falsity, as the case may be, finds its expression in the very sign for the proposition. I have not yet succeeded in finding a notation for identity that satisfies this condition; but I have NO doubt that it must be possible to find such a notation. For compound propositions ("elementary propositions") the ab-notation is sufficient. (Wittgenstein (1995), p. 60)

(See Lampert (2017) for recent discussion of the ab-notation.) Wittgenstein never acknowledges in Tractatus that he departs from Russell and Whitehead's usage; given the 1913 letter and Principia's authority, one might wonder why. By the time of Tractatus, Wittgenstein is convinced that 'all propositions are results of truth-operations on the elementary propositions' (5.3) while, in turn, elementary propositions are uncompounded. So the distinction between truth-functional compounds and quantified propositions entirely natural in the context of the theory of types - collapses, and the label 'elementary proposition', which before marked one side of the distinction, is available for other use. By 1919 Wittgenstein takes his redeployment of the label for granted: 'Of course no elementary props are negative' (letter to Russell, in Wittgenstein (1995) p. 126). When Ramsey later reverts to Russell's usage, he is careful to note this fact (Ramsey (1926), footnote on p. 341). Nowhere known to me do these authors suggest that a proposition might be truth-functionally compound or simple in a relative sense. 
But while the subject is beyond positive description, 'there remains the reality coordinated with it' (5.64), and this we can positively characterize. Coordination between subject and reality is effected via language, whose limits coincide with reality's:

That the world is my world, shows itself in the fact that the limits of the language (the language which I understand) mean [bedeuten] the limits of my world. (5.62)

At the limits of language sit elementary propositions, and what the simple parts of elementary propositions refer to - 'the limits of $m y$ world' - are objects:

Empirical reality is limited by the totality of objects. The boundary appears again in the totality of elementary propositions. (5.5561)

If an elementary proposition is false then the atomic fact that would exist if the proposition were true does not exist, but the objects named in the elementary proposition exist in any case (2.014). This is why the limits of language mean objects rather than atomic facts.

We come now to the sense in which objects are dependent for their simplicity on the subject. Wittgenstein states directly how the limits of language are settled: ' $[\mathrm{t}] \mathrm{he}$ application [Anwendung] of logic decides what elementary propositions there are' (5.557). Wittgenstein uses the word Anwendung mostly in connection with logical operations, as when he writes that 'truth-functions are results of the successive application of a finite number of truth-operations to elementary propositions' (5.32). On this usage, both the logical analysis of a proposition and the buildup of logical space by truth-functionally compounding the proposition with other propositions are applications of logic. But there is a further connection in which Wittgenstein speaks of application:

The name cannot be analysed further by any definition. It is a primitive sign. (3.26)

What does not get expressed in the sign is shown by its application. What the signs conceal, their application declares. (3.262)

These uses of the term Anwendung prompt two questions. First, how does the application of logic 'decide' what elementary propositions there are? Second, is it the same sort of application that 'declares' a sign to be primitive?

Wittgenstein addresses both questions at one go in entries that predate by a few days the exclamation quoted by Moss. The entries call for reproduction in full:

[W] hat we mean by "complex objects do not exist" is: It must be clear in the proposition how the object is composed, so far as it is possible for us to speak of its complexity at all.- The sense of the proposition must appear in the proposition as divided into its simple components-. And these parts are then actually indivisible, for further divided they just would not be THESE. In other words, the proposition can then no longer be replaced by one that has more components, but any that has more components also does not have this sense. 
When the sense of the proposition is completely expressed in the proposition itself, the proposition is always divided into its simple components-no further division is possible and an apparent one is superfluous - and these are objects in the original sense. (17.6.15)

But suppose that a simple name denotes an infinitely complex object? For example, perhaps we assert of a patch in our visual field that it is to the right of a line, and we assume that every patch in our visual field is infinitely complex. Then if we say that a point in that patch is to the right of the line, this proposition follows from the previous one, and if there are infinitely many points in the patch then infinitely many propositions of different content follow LOGICALLY from that first one. And this of itself shews that the proposition itself was as a matter of fact infinitely complex. That is, not the propositional sign by itself, but it together with its syntactical application [Verwendung]. (18.6.15)

A sign is structured in accordance with the inferences licensed by its occurrence. To declare 'the patch' complex in 'the patch is to the right of the line' is to decide that the region of logical space occupied by the proposition requires more than one parameter for its specification. The smaller the region, the larger the number of regions that contain it, that is, the more it entails 13 Whether an expression is truth-functionally compound or elementary has nothing directly to do with whether it displays a logical constant - its 'apparent simplicity' - but everything to do with its application.

Let us consider an example. Recall the analysis of wedded/single space in $\$ 2$. $a$ is wedded just in case $a$ is married and single just in case $a$ is not married. As I noted there, the analysis proceeds in accordance with definitions in my idiolect. Further definitions, fixed by my 'syntactical application' of the relevant expressions, will decide whether ' $a$ is married' is elementary or compound. In accordance with the principle of unique logical analyzability, the proposition will vary with the analysis. Which proposition I settle on is subject to no external constraint but is definitional and immediately given ('this sense'). The identity of logical form in a proposition and in the fact that it describes entails that any idiolect that would model another will assimilate the other, up to superficial differences of sign (4.025). The relativization of objects to the subject thus passes over into solipsism. Solipsism might be said to narrow the scope of the color incompatibility problem but it does not support the relativity thesis.

\footnotetext{
13 Wittgenstein hints in the notebooks at an information-theoretic interpretation of volume in logical space:

For what I should now have to do is to find an expression in the language of this theory for HOW MUCH a proposition says. And this would have to yield the result that tautologies say NOTHING. But how can we find the measure of amount-that-is-said? (2.6.15)

One could certainly say: That proposition says the most from which the most follows. (3.6.15)

A similar notion is glimpsed in Frege (1980), where Frege comments that 'the content of a concept diminishes as its extension increases' (§29). This notion coincides with neither sinn nor bedeutung, which Frege distinguished only in later work. Frege does not bring the notion to bear on the concept $x \neq x$ when he uses $x \neq x$ to define 0 (§74). One naturally wonders if Wittgenstein's information-theoretic idea was inspired by Frege's comment. Traces of the idea survive in Tractatus, e.g., in the account of conditional probability (5.15).
} 
Wittgenstein's notebooks seem to offer support of another kind to the relativity thesis. Wittgenstein was seized for a time by the idea that '[i]t must be through generality that ordinary propositions get their stamp of simplicity' (26.4.15). On this view, names generalize over facts concerning their bearers, as the name of a city might be thought to generalize over possible street plans (cf. 30.5.15). If this is so then names might introduce logical complexity, possibly even into propositions that nevertheless qualify as elementary.

Whatever its interest, this avenue to the relativity thesis is closed. The residue in Tractatus of Wittgenstein's early idea about names is a mostly unremarked duality between names and tautology. Names do not stand for facts (3.144), and neither does tautology (5.142). Tautology 'allows every possible state of affairs' (4.462), while objects, for which names go proxy, 'contain the possibility of all states of affairs' (2.014; cf. 2.021-2.024). 'The simple signs employed in propositions are called names' (3.2202), but tautologies, too, 'cannot be combinations of signs at all' (4.466). 'Names resemble points; propositions resemble arrows, they have sense' (3.144), but tautology, which is 'without sense', resembles both a 'substanceless centre' (5.143) and 'the point from which two arrows go out in opposite directions' (4.461). Like Blake's grain of sand, Tractarian names are world-encompassing. This gives no support to the relativity thesis. Tautology follows from everything and entails nothing but itself, in other words, it is logically idle. Recalling the close tie between structure and inference, one sees why Wittgenstein would go as far as to write that unfalsifiable expressions 'cannot be combinations of signs at all'.

There is a possible objection to the reading of Tractatus that I have been offering. Wittgenstein makes it clear that objects are meant to anchor propositions, conceived as expressions of truth-conditions by means of signs with unconditional reference:

If the world had no substance, then whether a proposition had sense would depend on whether another proposition was true. (2.0211)

It would then be impossible to form a picture of the world (true or false).

(2.0212)

According to Glock, Tractarian objects are supposed to be able to serve this function because they are 'indestructible' (Glock (1996) p. 269). If Wittgenstein relativizes objects to the subject as I have argued that he does, it makes no more sense for him to say that objects are indestructible than it does for him to say that they are destructible: they are conditions on sense decided by syntactical application, not items satisfying a description ('indestructible'). So, if Glock is right, and if the sense in which Tractarian objects are indestructible is just the sense in which, on my reading, they are neither indestructible nor destructible, then my reading is incorrect and the passages cited by Moss might yet support the relativity thesis.

Glock provides no direct textual evidence that Tractarian objects are indestructible, but Wittgenstein (2009) §46ff seems to promise the strongest case, albeit retrospective. In $\S 46$, Wittgenstein quotes Theaetetus to the effect that 'it is impossible to give an account of any primary element; for it, nothing is possible but the bare name'. Immediately he adds that 'Russell's "individuals" and my "objects" (Tractatus Logico-Philosophicus) were such primary elements'. In $\S 50$, Wittgenstein introduces the idea of indestructibility: 
What does it mean to say that we can attribute neither being nor non-being to elements?-One might say: if everything that we call "being" and "nonbeing" consists in the existence and non-existence of connexions between elements, it makes no sense to speak of an element's being (non-being); just as when everything that we call "destruction" lies in the separation of elements, it makes no sense to speak of the destruction of an element.

Wittgenstein does not say here that Tractarian objects were supposed to be indestructible. He dramatizes, in a metaphysical register, what in Tractatus he did maintain: 'it makes no sense to speak of an element's being (non-being)'. This way of dramatizing the Tractarian doctrine was not new in Philosophical Investigations: soon after his return to philosophy, Wittgenstein wrote that '[i]f someone says that substance is indestructible, then what he is really after is that it is senseless in any context to speak of "the destruction of a substance"-either to affirm or deny it' (Wittgenstein (1975) §95). Possibly this is the view that Glock wished to attribute to Wittgenstein. Be that as it may, there is no objection on grounds of the indestructibility of objects to the readings that I have offered, hence also no reason to suspect previously unrecognized commitments in Tractatus that might obviate the objections I have raised to Moss's proposal.

\section{Conclusion}

Haim Gaifman once emphasized in conversation with me that Wittgenstein's aim in Tractatus was philosophical rigor, not technical rigor. Contemporary discussion of the work sometimes focuses on technical details at the expense of philosophy. One ostensibly technical challenge that cannot be rightly understood absent a clear view of the work's philosophical underpinnings is the color incompatibility problem. A solution to the problem will be a logical analysis of color attributions resting stably on those underpinnings, among them:

(a) the dependence of the proposition on its logical analysis;

(b) the dependence, in turn, of logical space on elementary propositions;

(c) the relation between propositions and their negations;

(d) the difference between 'change of mesh' and logical analysis; and,

(e) the relativization of logical form to the subject via application.

These are the underpinnings on which I have focused in this paper.

My discussion has been guided throughout by Wittgenstein's comparison of propositions to measuring instruments ${ }^{14}$ Measurement is, one might quip, a model of the modeling relation that lies at the center of Wittgenstein's concern. This 'model of modeling' admits extension to a model of logical space and then again to a model of logical analysis. The quip goes lame, however, in suggesting that the relation between a Tractarian proposition and what it models might be subject to genuine modeling, that is to say, description. The relation between a Tractarian proposition and what

\footnotetext{
14 I am grateful to two anonymous reviewers of this journal for spurring me to the reflections in this and the following paragraphs.
} 
it models is no more a fact fit for description - no more a material relation - than Tractarian objects are indestructible. The comparison to measurement forms part of the ladder that Wittgenstein means his readers to kick away.

The kicking is to be done after climbing. Measurement boasts features that are seldom overlooked but whose analogues in thought - in 'projecting' possible states of affairs (3.11) - do easily slip from view. No fabric dealer forgets that the sum of two numbers is realized in the repeated controlled displacement of a rod relative to a stretch of fabric. Even a notion as abstract as the zero of a scale is without much ado understood concretely through the unit, so that no one boggles at the fact that, say, $0^{\circ} \mathrm{C} \neq 0^{\circ} \mathrm{F}$. When Wittgenstein compares propositions to measuring instruments, he is drawing attention to the concreta that realize logical space.

More specifically, the comparison to measurement illuminates each of (a) (e) and not only singly but in their intricate mutual relations. That classical force and momentum are different is shown by the equations $F=m a$ and $p=m v$, with $v$ having dimension length $\times$ time $^{-1}$ and $a$ having dimension length $\times$ time $^{-2}[15$ In order for a numerical magnitude to represent a force or momentum, base units must still be chosen, units that, in turn, are realized in the application of physical instruments. Similarly, the content of a point of logical space is fixed by the application of elementary propositions realized in signs. Thus (a) and (b) are figured together in the comparison to measurement, and with them (c) (e) the relation of a proposition to its negation, analogous to the relation between a scale and its zero; the possibility of changing 'meshes', analogous to the possibility of alternative coarse-grainings; and the relativization of logical space to the subject via application, analogous - with dramatic caveats - to the relativization of a physical system to the investigator via the choice of base quantities and units.

It is worth underscoring how evident the misreadings of Tractatus that we have considered in this paper become when examined in light of the comparison to measurement, or put another way, how much of the philosophical spirit of Tractatus the comparison captures. One might distinguish abstractly between a proposition and its negation and then assign them incompatible fragments of color space as their contents, but few will be tempted to stipulate the physical content of a scale's zero independently of the unit. Or again, one might imagine that a proposition's truthfunctional structure varies with one's choice of 'mesh', but one does not come naturally by the idea that the points in a region of phase space change according to one's choice of coarse-graining. Spotting one misunderstanding in light of the comparison to measurement, the others leap to view.

Moss's proposed solution to the color incompatibility problem fails, but I hope to have shown that serious engagement with the issues it raises conduces to deeper understanding of Tractatus and, thereby, to deeper insight into issues of contemporary interest in philosophy of logic.

Acknowledgements This paper is rooted in discussions concerning Tractatus that I was fortunate to have with Doron Avital over several years. Randall Havas and three anonymous reviewers caught many errors and helped me to frame key points. I began work on the paper under particularly favorable circumstances:

\footnotetext{
15 Acceleration and velocity are vector quantities while dimensional analysis works only on the magnitudes. We can ignore the additional information here at no cost in philosophical insight.
} 
as a Fulbright US Scholar hosted by the University of Social Sciences and Humanities (USSH) at Vietnam National University, Hanoi. I gratefully acknowledge the support of Fulbright, USSH, and also my home institution, Willamette University, which made it possible to accept the Fulbright award by granting me sabbatical leave. Among the colleagues and friends in Hanoi whose philosophical stimulation and hospitality nurtured this paper in its earliest stages, I owe special thanks to Professor Nguyễn Vũ Hảo of USSH and Hồ Mạnh Tùng of the Institute of Philosophy, Vietnam Academy of Social Sciences.

\section{References}

Carruthers, P. (1990). The Metaphysics of the Tractatus. Cambridge: Cambridge University Press.

Frascolla, P. (2007). Understanding Wittgenstein's Tractatus. New York: Routledge.

Frege, G. (1980). Foundations of Arithmetic. Evanston: Northwestern University Press.

Glock, H-J. (1996). A Wittgenstein Dictionary. Malden: Blackwell Publishers.

Lampert, T. (2017). Wittgenstein's ab-Notation: An iconic proof procedure. History and Philosophy of Logic, 38(3), 239-262.

Miller, H. (1995). Tractarian semantics for predicate logic. History and Philosophy of Logic, 16(2), $197-$ 215

Moss, S. (2012). Solving the color incompatibility problem. Journal of Philosophical Logic, 41(5), 841851

Ridderbos, K. (2002). The coarse-graining approach to statistical mechanics: How blissful is our ignorance? Studies in History and Philosophy of Science Part B: Studies in History and Philosophy of Modern Physics, 33(1), 65-77.

Rogers, B. and Wehmeier, K. F. (2012). Tractarian First-Order Logic: Identity and the N-operator. The Review of Symbolic Logic, 5(4), 538-574.

Ramsey, F. P. (1926). The Foundations of Mathematics. Proceedings of the London Mathematical Society, Series 2, 25(1), 338-384.

Russell, B. and Whitehead, A. N. (1910). Principia Mathematica. Cambridge: Cambridge University Press. Wittgenstein, L. (1929). Some Remarks on Logical Form. Supplementary Volume - Aristotelian Society, 9(1), 162-171.

Wittgenstein, L. (1961). Notebooks, 1914-1916. New York: Harper \& Brothers.

Wittgenstein, L. (1975). Philosophical Remarks. Chicago: University of Chicago Press.

Wittgenstein, L. (1979). Wittgenstein and the Vienna Circle. Oxford: Basil Blackwell

Wittgenstein, L. (1995). Wittgenstein: Cambridge Letters. Cambridge: Cambridge University Press.

Wittgenstein, L. (1998). Tractatus Logico-Philosophicus. Mineola, NY: Dover Publications.

Wittgenstein, L. (2009). Philosophical Investigations. Malden, MA: Wiley-Blackwell. 